\title{
AGER1 downregulation associates with fibrosis in nonalcoholic steatohepatitis and type 2 diabetes
}

\author{
Ali Dehnad, ${ }^{1}$ Weiguo Fan, ${ }^{1}$ Joy X. Jiang, ${ }^{2}$ Sarah R. Fish, ${ }^{2}$ Yuan Li, ${ }^{1}$ Suvarthi Das, ${ }^{1}$ Gergely Mozes, ${ }^{1}$ Kimberly A. Wong, ${ }^{3}$ \\ Kristin A. Olson, ${ }^{4}$ Gregory W. Charville, ${ }^{5}$ Mohammed Ali, ${ }^{6}$ and Natalie J. Török ${ }^{1}$
}

${ }^{1}$ Gastroenterology and Hepatology, Stanford University, Stanford, and VA Palo Alto, California, USA. ${ }^{2}$ Gastroenterology and Hepatology, ${ }^{3}$ Department of Internal Medicine, and ${ }^{4}$ Department of Pathology, UC Davis Medical Center, Sacramento, California, USA. 5Department of Pathology, Stanford University, Stanford, California, USA. ${ }^{6}$ Department of Surgery, UC Davis Medical Center, Sacramento, California, USA.

\begin{abstract}
Type 2 diabetes is clinically associated with progressive necroinflammation and fibrosis in nonalcoholic steatohepatitis (NASH). Advanced glycation end-products (ACEs) accumulate during prolonged hyperglycemia, but the mechanistic pathways that lead to accelerated liver fibrosis have not been well defined. In this study, we show that the AGEs clearance receptor AGER1 was downregulated in patients with NASH and diabetes and in our NASH models, whereas the proinflammatory receptor RAGE was induced. These findings were associated with necroinflammatory, fibrogenic, and pro-oxidant activity via the NADPH oxidase 4. Inhibition of AGEs or RAGE deletion in hepatocytes in vivo reversed these effects. We demonstrate that dysregulation of NRF2 by neddylation of cullin 3 was linked to ACER1 downregulation and that induction of NRF2 using an adeno-associated virus-mediated approach in hepatocytes in vivo reversed AGER1 downregulation, lowered the level of AGEs, and improved proinflammatory and fibrogenic responses in mice on a high AGEs diet. In patients with NASH and diabetes or insulin resistance, low ACER1 levels were associated with hepatocyte ballooning degeneration and ductular reaction. Collectively, prolonged exposure to AGEs in the liver promotes an AGER1/RAGE imbalance and consequent redox, inflammatory, and fibrogenic activity in NASH.
\end{abstract}

\section{Introduction}

Nonalcoholic steatohepatitis (NASH) is rapidly becoming the most common chronic liver disease worldwide. Patients with insulin resistance or type 2 diabetes mellitus (T2DM) are at risk for developing more advanced NASH with necroinflammation and fibrosis progression $(1,2)$. Hyperglycemia in diabetes predisposes individuals to the formation and accumulation of advanced glycation end-products (AGEs) $(3,4)$. AGEs are either produced by the Maillard reaction or nonenzymatic glycation of serum proteins (5), or could be formed by reaction with glyoxal, methylglyoxal, or 3-deoxyglucosone as a result of consuming foods prepared at high temperatures. These highly reactive compounds elicit inflammatory reactions and contribute to vascular, renal, or retinal complications in $\mathrm{T} 2 \mathrm{DM}(6,7)$. It is very plausible that AGEs are instrumental in pathways that lead to necroinflammation and fibrosis in NASH; however, the mechanistic aspects of AGE-mediated injury or the liver cell types that are involved have not been clearly identified. AGEs are removed from the circulation and detoxified by the AGE receptor 1 (AGER1) $(8,9)$. The role of AGER1 in NASH has not been studied. It is plausible that impaired AGER1 expression or function during conditions of high AGEs in patients with diabetes could lead to further accumulation of AGEs and consequent engagement of the receptor for AGEs (RAGE) with subsequent proinflammatory responses (10).

Conflict of interest: The authors have declared that no conflict of interest exists. Copyright: () 2020, American Society for Clinical Investigation.

Submitted: August 29, 2019; Accepted: May 5, 2020; Published: July 13, 2020.

Reference information: J Clin Invest. 2020;130(8):4320-4330.

https://doi.org/10.1172/JCI133051.
On the other hand, RAGE is linked to the production of oxidative radicals by activation of the nicotinamide adenine dinucleotide phosphate (NADPH) oxidases (NOXs). It remains to be clarified which NOX isoform is involved in generating ROS in response to AGEs/RAGE engagement in the liver.

\section{Results}

Critical role of hepatocyte RAGE in modulating inflammation and fibrosis during a high-AGEs diet. Hepatocytes express RAGE; however, its function in these cells has not been well studied. To focus on AGEs and RAGE, we used a high-AGEs diet, as the current NASH diets for animal studies are not particularly high in AGE content. We prepared a diet high in AGEs (HiAD) at high temperature and fed mice this diet for 14 weeks. Serum and liver AGEs in mice on this diet were higher than those in mice on a fast food diet (FFD) that is commonly used to study NASH (11) (Figure 1A, Supplemental Figure 1, and diet composition is included in Supplemental Table 1; supplemental material available online with this article; https://doi.org/10.1172/JCI133051DS1). We treated a group of $f l / f l$ Rage mice (hereafter referred to as WT mice) on a HiAD with pyridoxamine (PM), a vitamin $\mathrm{B} 6$ derivative that has been shown to reduce AGEs by trapping the carbonyl compounds and inhibiting the formation of Amadori products, and therefore AGE formation $(12,13)$, or vehicle. We also generated RAGE hepatocyte-KO $\left(\right.$ Rage $\left.^{\text {HepKO }}\right)$ mice and placed them on a chow diet or a HiAD. These mice had a normal phenotype on the chow diet. We found that liver and serum AGEs decreased HiAD-fed WT mice treated with PM as well as in HiAD-fed Rage ${ }^{\text {HерКO }}$ mice (Figure 1B). In WT mice on a HiAD, we observed severe NASH with necroinflammation, hepatocyte ballooning, steatosis (NAFLD 

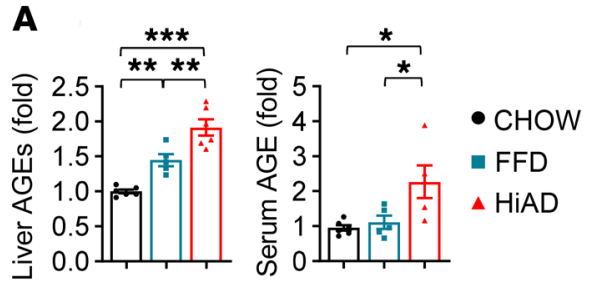
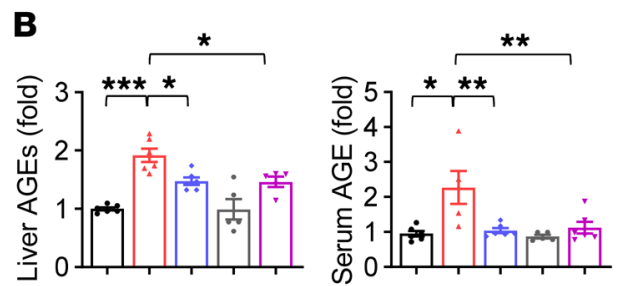

- WT CHOW

$\triangle W T H A D$

- WT HiAD + PM

- Rage ${ }^{\text {HepKO }} \mathrm{CHOW}$

$\checkmark$ Rage ${ }^{\text {Hepko }}$ HiAD
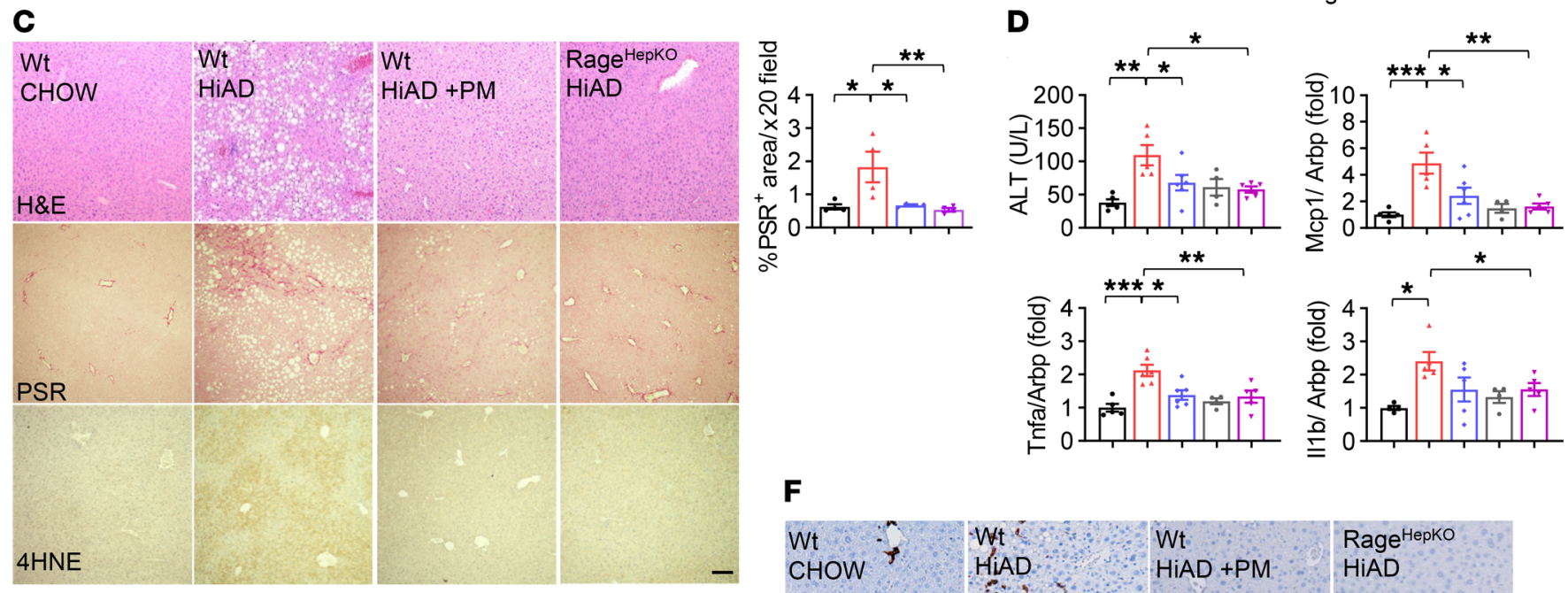

$\mathbf{F}$
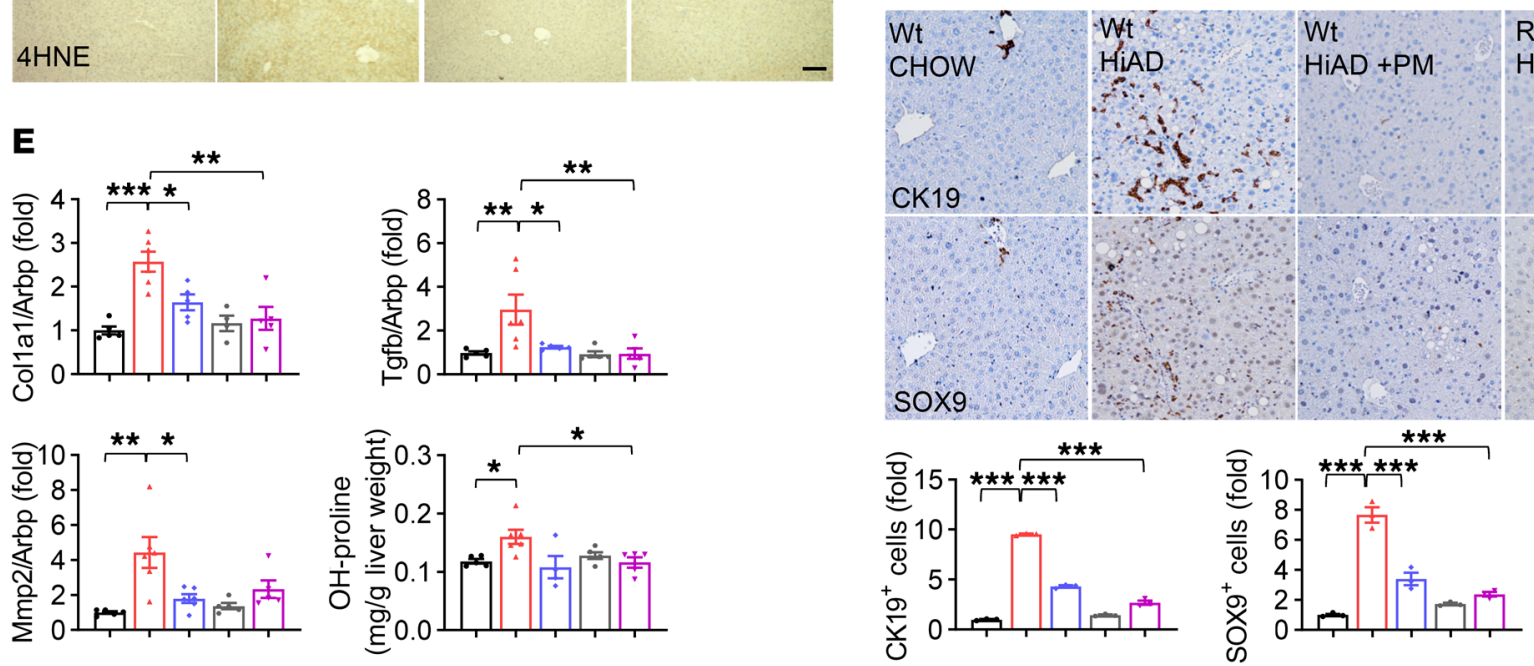

Figure 1. Hepatocyte RAGE is required for proinflammatory and fibrogenic signals in mice on a HiAD. (A) A HiAD induced a more significant increase

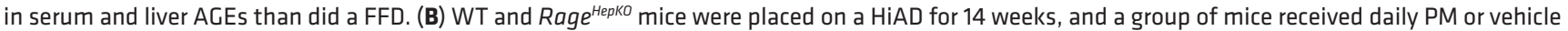
(Tris-HCI). Liver AGEs increased in WT mice on a HiAD and decreased in these mice following PM treatment as well as in Rage ${ }^{\mathrm{HepKO}}$ mice on a HiAD. Serum AGEs markedly increased with a HiAD, and this was attenuated by treatment with PM, whereas no increase was seen in Rage $e^{\text {Hepko }}$ mice on a HiAD. (C) H\&E-stained images show inflammation and steatosis in HiAD-fed WT mice that improved after PM treatment and in HiAD-fed Rage ${ }^{\text {HepKO }}$ mice. NAS

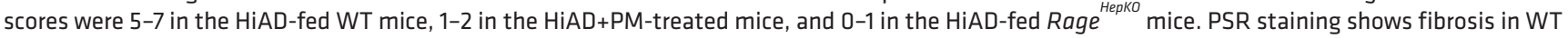
mice on a HiAD, with improvement after PM treatment, and in Rage ${ }^{\text {HepKo }}$ mice on a HiAD. PSR staining was analyzed with Imagej software. Collagen deposition was lower in the HiAD+PM and Rage ${ }^{H e p k O}$ groups ( $n=4$ mice/group, 4 random $\times 20$ fields/sample; data are presented as the percentage of

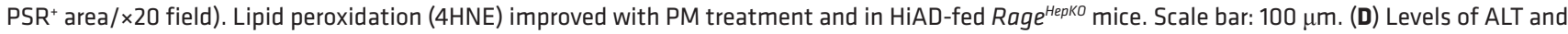
inflammatory transcripts Mcp1, Tnfa, I/1b were reduced in PM-treated and Rage ${ }^{\text {HepKO }}$ mice. (E) Levels of fibrosis-related transcripts Col1a1, Tgfb, Mmp2 and hydroxyproline ( $\mathrm{OH}$-proline) decreased in PM-treated and Rage ${ }^{\text {HepKO }}$ mice. (F) CK19+ and SOX9+ RDCs were observed in the HiAD-fed mice. The number of positive cells was lower in the PM-treated mice and in Rage ${ }^{\text {Hерко }}$ mice on a HiAD. Scale bar: $100 \mu \mathrm{m}$. Images for FFD data are shown in Supplemental Figure 5. Data represent the mean $\pm \mathrm{SEM} .{ }^{*} P<0.05,{ }^{* *} P<0.01$, and ${ }^{* * *} P<0.001$, by 1 -way ANOVA followed by post hoc Tukey's test.

activity score [NAS] of 5-7), fibrosis (Picrosirius red [PSR]), and lipid peroxidation (4HNE) (Figure 1C). Body weight and liver/ body weight ratios were not significantly different in the treatment groups (although the HiAD group had a trend toward lower weights because of smaller initial food intake, Supplemental Figure 2). ALT levels, NAS scores (1-2 in the HiAD+PM group; $0-1$ in
Rage ${ }^{\text {HерKO }}$ group), the expression of inflammation transcripts (Mcp1, Tnfa, Il1b) (Figure 1D), and the expression of fibrogenic transcripts (Col1a1, Tgfb1, Mmp2) were significantly attenuated in WT mice on a HiAD treated with PM and in Rage ${ }^{\text {HepKO }}$ mice on a HiAD (Figure 1E). Hydroxyproline content decreased in PM-treated WT and Rage ${ }^{\text {HepKO }}$ mice on a HiAD (Figure 1E). Interestingly, we found 
A

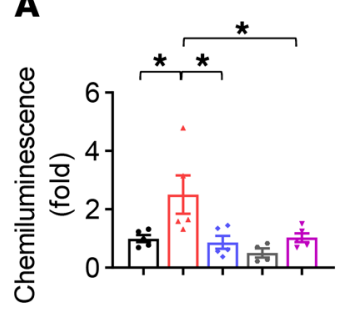

D

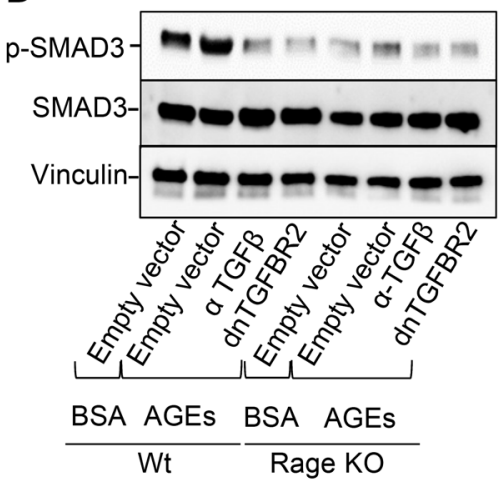

B

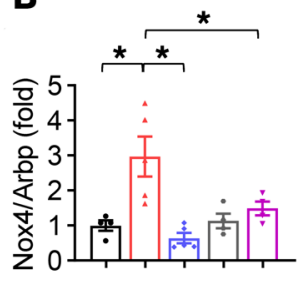

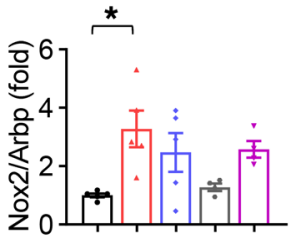

C
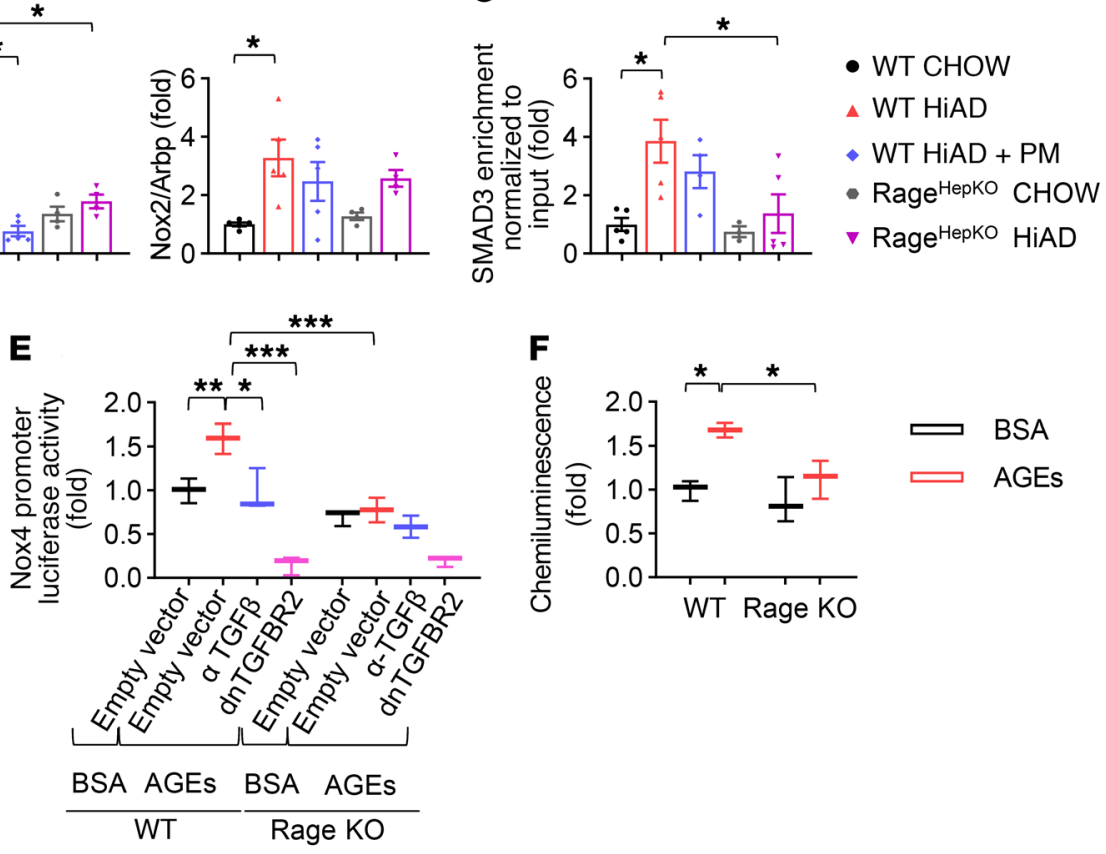

Figure 2. RAGE and NOX4 mediate oxidative injury in hepatocytes by AGEs. (A) ROS production was measured by lucigenin chemiluminescence. HiAD-

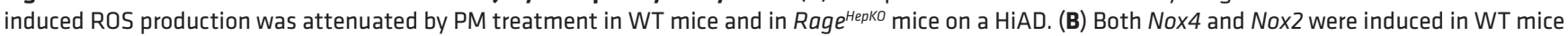
on a HiAD, but only Nox4 induction was attenuated by PM treatment and in Rage ${ }^{\text {HepKo }}$ mice on a HiAD. (C) ChIP was performed to study Nox4 promoter induction. Significant SMAD3-dependent induction was seen in WT mice on a HiAD, and this was reduced in these mice with PM treatment as well as in Rage Нерко mice on a HiAD. (D) Primary WT or Rage-KO hepatocytes were treated with $100 \mu \mathrm{g} / \mathrm{mL}$ ACEs-BSA, in the presence or absence of TCF- $\beta$ neutralizing antibody ( $\alpha-\mathrm{TGF}-\beta, 5 \mu \mathrm{g} / \mathrm{mL}$ ), or were transfected with dn-TGF- $\beta R 2$ or an empty plasmid. SMAD3 phosphorylation ( $\mathrm{p}-\mathrm{SMAD3}$ ) was attenuated by the TGF- $\beta$ antibody or by transfection of dn-TGF- $\beta$ R2, and no induction of p-SMAD3 was seen in Rage-KO cells. (E) WT and Rage-KO primary mouse hepatocytes were treated with $100 \mu \mathrm{g} / \mathrm{mL}$ AGEs-BSA for 24 hours. The Nox4 luciferase promoter assay indicated AGE-mediated Nox4 promoter activity that was reduced in Rage-KO cells and in those exposed to anti-TGF- $\beta$ or transfected with the dn-TCF- $\beta$ R2. (F) ROS production increased in ACE-exposed WT hepatocytes, and this was attenuated in Rage-KO cells, as shown by chemiluminescence assay. Data in $\mathbf{A}-\mathbf{C}$ represent the mean \pm SEM. Data in $\mathbf{E}$ and F were combined from 3 replicates. Bars indicate the 25th-75th percentiles, lines indicate the median, and whiskers indicate minimum and maximum values. ${ }^{*} P<0.05,{ }^{*} P<0.01$, and ${ }^{* *} P<0.001$, by 1 -way ANOVA with Tukey's post hoc test.

that alkaline phosphatase showed an increasing trend in WT mice on a HiAD that improved after PM treatment, and the same was observed in Rage ${ }^{\mathrm{HepKO}}$ mice on a HiAD. We observed no significant change in total bilirubin levels (Supplemental Figure 3). Insulin sensitivity in WT mice on a HiAD was impaired but improved in Rage ${ }^{\mathrm{HePKO}}$ mice on a HiAD as assessed by glucose tolerance test/ insulin tolerance test (GTT/ITT) (Supplemental Figure 4).

Several studies have highlighted the link between the appearance of reactive ductular cells (RDCs) and fibrosis in NASH (14, 15). We observed an expansion of $\mathrm{CK} 9^{+}$and SOX9+ $9^{+} \mathrm{RD}$ in the $\mathrm{HiAD}$-fed mice, whereas expansion was much lower in the mice on a FFD (which has lower AGE content; Supplemental Figure 5). We detected significantly lower numbers of RDCs in the PM-treated mice and in Rage ${ }^{\mathrm{HepKO}}$ mice on HiAD (Figure 1F).

AGEs induce NOX4 and ROS production in hepatocytes via RAGE. 4HNE had an intense signal in hepatocytes from mice on a

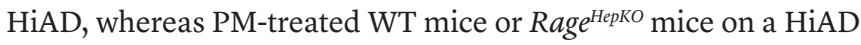
had significantly less lipid peroxidation, as shown earlier (Figure 1C). Lucigenin chemiluminescence revealed decreased production of ROS in PM-treated WT mice and Rage ${ }^{\text {HерКО }}$ mice on a HiAD (Figure 2A). NOX4, a transcriptionally activated nonphagocytic NOX homolog in hepatocytes, is a major source of redox radicals in NASH and is closely linked to ER stress, hepatocyte death, and fibrogenic activity (16). We observed reduced Nox4 expression in PM-treated WT mice and Rage ${ }^{\mathrm{HepKO}}$ mice on HiAD (Figure 2B), whereas Nox2 (phagocytic NOX) was induced in mice on a HiAD but did not change significantly in PM-treated WT mice or in Rage $e^{\text {HepKO }}$ mice on a HiAD. This posed an interesting question of whether AGEs and RAGE target NOX4 as a downstream effector. To address this question, we performed ChIP assays to study Nox4 promoter activation in our animal models. Real-time quantitative PCR (RT-qPCR) analysis of SMAD3-enriched DNA revealed significantly higher Nox4 induction in liver samples from mice on a HiAD that was reduced in the HiAD-fed Rage $e^{\text {HepKO }}$ mice (Figure 2C). To address the mechanism of NOX4 induction in primary hepatocytes, AGE-exposed cells were treated with either a TGF- $\beta$-neutralizing antibody or transfected with dominantnegative TGF- $\beta$ R2 (dn-TGF- $\beta$ R2) versus empty vector, and SMAD3 phosphorylation (p-SMAD3) was assessed (Figure 2D). AGEs induced SMAD3 phosphorylation, and this was reduced by the TGF- $\beta$ antibody or dn-TGF- $\beta$ R2. Rage-KO hepatocytes did not exhibit SMAD3 phosphorylation after exposure to AGEs. Promoter assays in primary hepatocytes in the aforementioned conditions revealed induction of the Nox4 promoter and that AGEmediated TGF- $\beta$ indeed played an important, but not exclusive, role (Figure 2E). As observed in vivo, Rage-KO hepatocytes did 
A

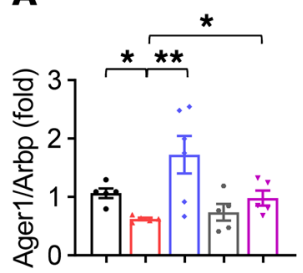

\author{
- WT CHOW \\ $\triangle W T$ HIAD \\ - WT HiAD + PM \\ - Rage ${ }^{\text {HepKO }} \mathrm{CHOW}$ \\ - Rage ${ }^{\text {Hepko }}$ HiAD
}
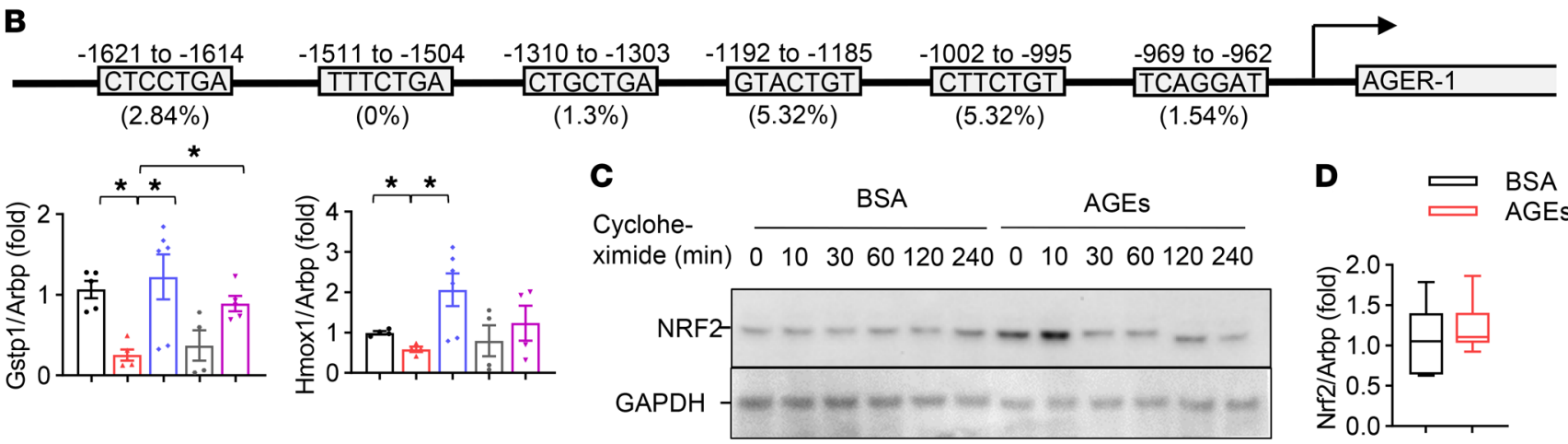

Figure 3. Ager1 is downregulated in HiAD-fed WT but not HiAD-fed Rage ${ }^{\text {HepKo }}$ mice. (A) Expression of Ager1, the clearance receptor for AGEs, was downregulated in WT mice on a HiAD, and this was reversed by the AGEs inhibitor PM and in Rage ${ }^{\text {HepKo }}$ mice on a HiAD. (B) The AGER1 promoter region (-3302 bp) was analyzed using ALGGEN-PROMO. Of the predicted transcriptional activators, NRF2 had 6 binding sites with less than $10 \%$ dissimilarity (shown as position and sequence dissimilarity). To study the role of NRF2 in AGER1 regulation, the NRF2 targets Gstp1 and Hmox1 were evaluated. Gstp1 and Hmox1 expression was reduced in mice on a HiAD, and this was reversed by PM treatment and in Rage ${ }^{\text {Hepko }}$ mice on HiAD. (C) To address NRF2 stability, primary WT hepatocytes were treated with AGEs (or control BSA) for 24 hours. Cells were incubated with cycloheximide ( $0.5 \mu \mathrm{g} / \mathrm{mL}$ ), and although initially there was an increase in NRF2, this was lost by prolonged exposure to AGEs (a representative Western blot from 3 independent experiments is shown). (D) RT-qPCR analysis revealed no significant change in Nrf2 mRNA expression in primary WT hepatocytes exposed to BSA or AGEs. Data in $\mathbf{A}$ and $\mathbf{B}$ represent the mean \pm SEM. ${ }^{*} P<0.05$ and ${ }^{*} P<0.01$, by 1-way ANOVA with Tukey's post hoc test. Data in $\mathbf{D}$ were combined from 5 replicates; bars indicate the 25th-75th percentiles, lines indicate the median, and whiskers indicate minimum and maximum values.

not exhibit ROS production following exposure to AGEs (Figure $2 \mathrm{~F})$. We further evaluated the signaling crosstalk that is activated by AGEs/RAGE and could be involved in the SMAD3/NOX4 axis. P38MAPK and JNK1/-2 phosphorylation was dependent on the presence of RAGE, and their inhibition reduced SMAD3 phosphorylation (Supplemental Figure 6).

To study the NOX homologs responsible for ROS production after exposure to AGEs, we isolated primary hepatocytes, hepatic stellate cells (HSCs), and macrophages from WT, Nox4-KO, and Nox2-KO livers (Supplemental Figure 7A) and exposed them to AGEs. In hepatocytes, NOX4 was the dominant NOX, whereas in HSCs, both NOX4 and NOX2, and in macrophages, mainly NOX2, played a role in AGE-mediated ROS production. However, in HSCs, AGEs did not directly induce transdifferentiation (Supplemental Figure 7B). To study this in vivo, we fed WT mice chow or a HiAD for 14 weeks, and at week 7, they were injected with adeno-associated virus type 6-Cre (AAV6-Cre) or control (AAV6-GFP). AAV6 has been described as targeting HSCs (17). Compared with AAV6-GFP-injected mice, we observed was no significant reduction in aSMA or Col1a1 or serum alanine aminotransferase (ALT) levels in mice on a HiAD (Supplemental Figure 7C). WT macrophages showed mild proinflammatory activity after exposure to AGEs, with decreased Tnfa, Il1O, and Illb production in Nox4- and Nox2-KO cells (Supplemental Figure 7D). To address the potential paracrine effects of AGE-stimulated hepatocytes, cells from WT and Nox4-KO mice were isolated and treated with $100 \mu \mathrm{g} / \mathrm{mL}$ AGEs, with or without $10 \mathrm{mM}$ glutathione (GSH). After 24 hours, the medium was changed, and using a Transwell coculture system, WT HSCs or macrophages were cocultured with hepatocytes (Supplemental Figure 8). We found that AGEexposed hepatocytes significantly induced aSMA and Col1a1 in WT HSCs. Use of GSH or Nox4-deleted hepatocytes in coculture significantly reduced HSC activation. In macrophages, Il1b, Tnfa, and $I l 6$ expression was significantly induced after coculture with AGE-exposed WT, by not Nox4-KO, hepatocytes. GSH treatment attenuated the expression of proinflammatory transcripts (Supplemental Figure 8).

Downregulation of AGER1 in hepatocytes is associated with decreased NRF2 activity. We studied AGER1 in our animal models and found that it was significantly downregulated in HiADfed mice (Figure 3A) and also in patients with NASH and T2DM (as shown later). In mice, treatment with PM augmented AGER1 expression, with a marked increase in Rage ${ }^{\mathrm{HepKO}}$ mice on a HiAD. To investigate the mechanism of downregulation, we analyzed the AGER1 promoter region using ALGGEN-PROMO (18), which revealed several NRF2-binding sites (Figure 3B). NRF2 nuclear translocation modulates downstream antioxidant response elements (AREs) in target genes, thereby attenuating oxidative stress (19-21). NRF2 targets Hmox1 and Gstp1 were significantly reduced in WT mice on a HiAD, and this was improved in these mice following PM treatment and in Rage ${ }^{\mathrm{HepKO}}$ mice on a HiAD (Figure 3B). Furthermore, the nuclear NRF2 signal was reduced 

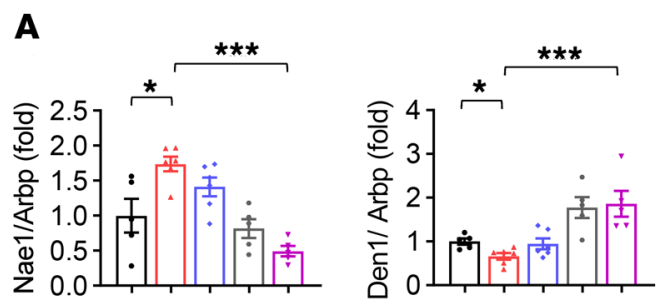

- WT CHOW

$\triangle$ WT HIAD

- WT HIAD + PM

- Rage ${ }^{\text {HepKO }} \mathrm{CHOW}$

- Rage ${ }^{\text {HepKO }} \mathrm{HiAD}$

B

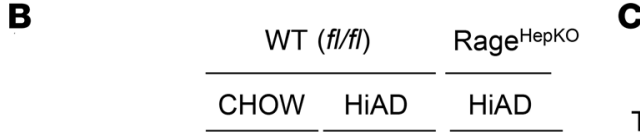

C

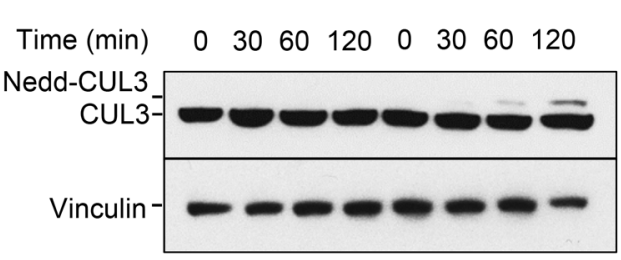

D

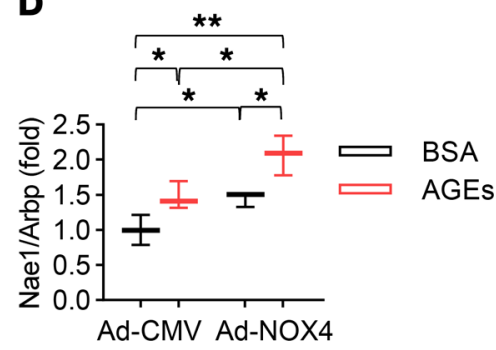

\section{$\mathbf{E}$}
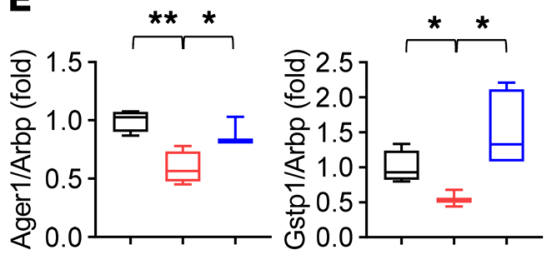
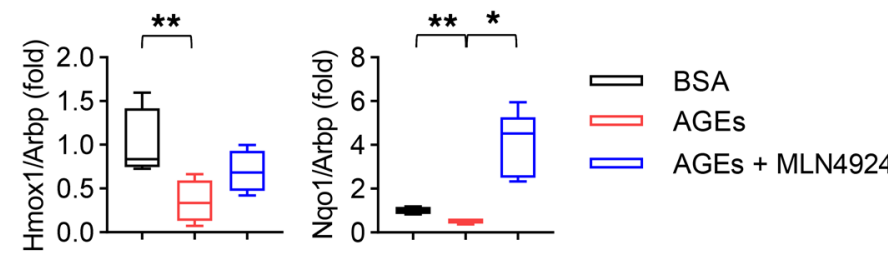

Figure 4. NRF2 activity is decreased by AGEs as a result of CUL3 neddylation. (A) Expression of Nae1 was increased in WT mice on a HiAD and significantly reduced in Rage ${ }^{\text {HepKO }}$ mice, whereas the deneddylase Den1 was reduced by a HiAD and reversed in Rage ${ }^{\text {HepKo }}$ mice. (B) Liver homogenates from WT or Rage ${ }^{\text {Hерко }}$ mice on a chow or HiAD were tested for CUL3, and immunoblotting revealed increased neddylation (Nedd) in WT mice on a HiAD, whereas Rage ${ }^{\text {HepKO }}$ mice were protected. (C) Time course of CUL3 neddylation by AGEs in HepG2 cells. (D) To determine whether NOX4 is involved in regulating NAE1 expression, primary hepatocytes were transduced with Ad-Nox4 or a control vector and after 48 hours were treated with $100 \mathrm{mg} / \mathrm{mL}$ AGEs or control BSA for 30 minutes. Expression of Nae1 was increased by NOX4 and exposure to AGEs. (E) The NAE1 inhibitor MLN4924 ( $3 \mu$ M) in hepatocytes reversed the effects of AGEs on Ager1, Gstp1, Hmox1, and Nqo1 expression. Data in A indicate the mean \pm SEM; date in $\mathbf{D}$ were combined from 3 replicates; data in $\mathbf{E}$ were combined from 4-5 replicates. In $\mathbf{D}$ and $\mathbf{E}$, bars indicate the 25 th-75th percentiles, lines indicate the median, and whiskers indicate minimum and maximum values. ${ }^{*} P<0.05,{ }^{* *} P<0.01$, and ${ }^{* *} P<0.001$, by 1-way ANOVA with Tukey's post hoc test $(\mathbf{A}, \mathbf{D}$, and $\mathbf{E})$.

in mice on a HiAD (Supplemental Figure 9). To study the potential role of NRF2 dysregulation in response to AGEs, the NRF2 stability assay in hepatocytes demonstrated early induction by AGEs, however, this was lost following more prolonged exposure, suggesting that AGEs promote increased degradation of NRF2 (Figure 3C). Nrf 2 mRNA expression was not significantly different between the BSA- and AGE-treated groups (Figure 3D).

NRF2 dysregulation is due to neddylation of the cullin 3 complex. NRF2 stability is regulated by complex mechanisms at multiple levels (22). In our case, experimental evidence pointed to posttranslational modification in response to AGEs. Cullin 3 (CUL3) serves as a scaffolding protein that is bound to both RBX1 and KEAP1 adaptor proteins, which attach to NRF2. Under oxidative stress, the ability of the CUL3-KEAP1-E3 ligase to ubiquitinate NRF2 is inhibited, which allows NRF2 to translocate to the nucleus. However, upon posttranslational modifications of CUL3, such as neddylation, this cannot occur and thus results in decreased NRF2 activity (22). We show that, in vivo, expression of NEDD8activating enzyme (Nael) was increased by a HiAD and was significantly reduced in Rage ${ }^{\mathrm{Hep} K O}$ mice on a HiAD, whereas expression of the deneddylase Den1 was induced by PM treatment and in Rage ${ }^{\mathrm{HepKO}}$ mice on HiAD (Figure 4A). CUL3 neddylation was apparent in WT mice on a HiAD but not in Rage ${ }^{\mathrm{HepKO}}$ mice (Figure 4B).
In vitro, exposure to AGEs resulted in the neddylation of CUL3 in HepG2 cells (Figure 4C). To explore whether NOX4 is involved in the activation of NAE1, we transduced primary hepatocytes with Ad-Nox4 and assessed Nae1 expression. We found that Ad-Nox4 or AGEs induced the enzyme and detected a more significant induction when Ad-Nox4 and exposure to AGEs were combined (Figure 4D). We evaluated the effect of neddylation on Ager1 and NRF2 target enzymes in the presence of AGEs and the NAE1 inhibitor MLN4924. Inhibition of neddylation resulted in a significant increase in the expression of Ager1, Gstp1, Hmox1, and Nqo1 (Figure 4E).

Correcting NRF2 by an AAV8-mediated approach improves AGER1, liver/serum AGEs, inflammation, and fibrosis. To determine whether lower NRF2 activity is linked to an inadequate AGER1 response, we injected mice on a HiAD at week 7 of the 14 -week diet with either AAV8-GFP (control) or AAV8-Nrf2. Downregulation of Ager1 was reversed in vivo by AAV8-Nrf2, and liver and serum AGEs were decreased (Figure 5A). These mice exhibited increased levels of Ager1 and the NRF2 target genes Gstp1 and Hmox1 (Figure 5B). Expression levels of transcripts for inflammation (Mcp1, Tnfa, Il1b) (Figure 5C) and fibrosis (Col1a1 and Tgfb) (Figure 5D) were reduced as well. Similarly, Ager1 was significantly downregulated by AGEs in primary WT hepatocytes, and adeno- 
A
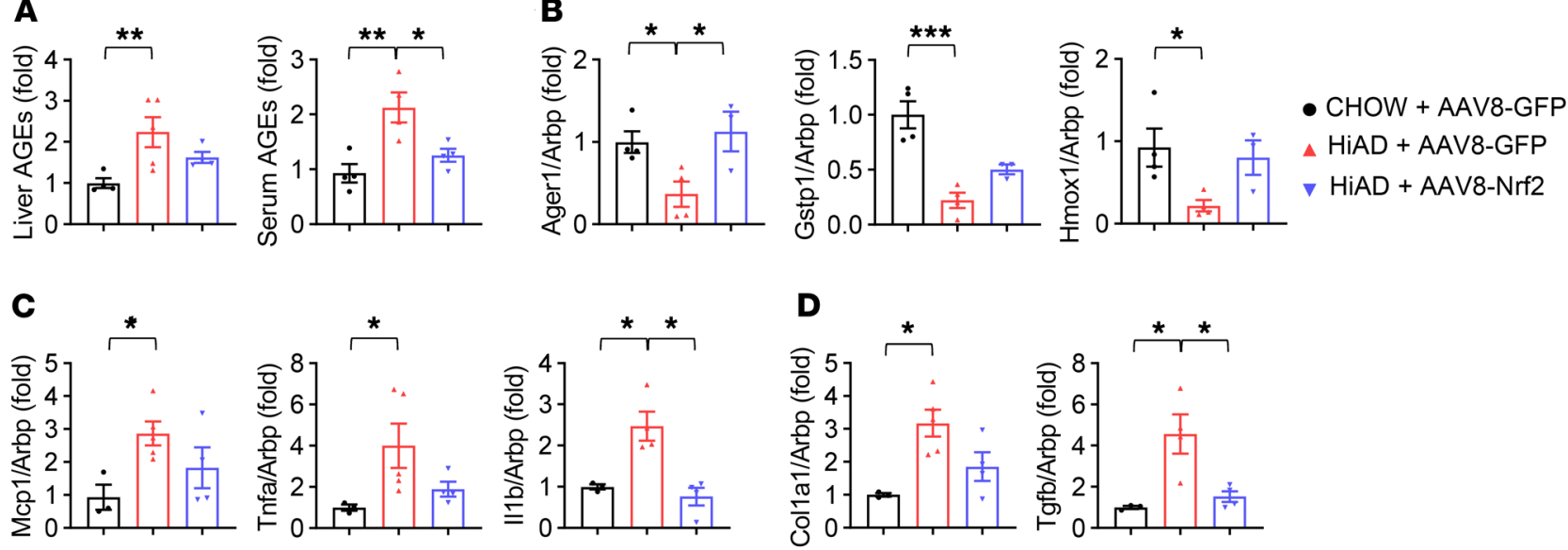

Figure 5. AAV8-mediated NRF2 transduction reduces the levels of hepatic and serum AGEs, Ager1, inflammation, and fibrosis in mice on a HiAD. (A) WT mice were fed a HiAD for 14 weeks. At week 7, a group of mice were injected with AAV8-TBG-Nrf2 ( $5 \times 10^{11} \mathrm{GC} / \mathrm{mouse}$ ) or AAV8-GFP as a control. There was a decrease in serum and liver AGEs in the AAV8-Nrf2-injected group. (B) Expression of Ager1 and the NRF2 targets Gstp1 and Hmox1 increased following AAV8-Nrf2 injection. (C) Expression of transcripts reflecting inflammation - Mcp1, Tnfa, and I/1b - was also significantly reduced. (D) Expression of Col1a1 and Tgfb was significantly decreased by Nrf2 transduction. Data are presented as the mean $\pm \mathrm{SEM}$. ${ }^{*} P<0.05,{ }^{* *} P<0.01$, and ${ }^{* * *} P<0.001$, by 1-way ANOVA with Tukey's post hoc test.

viral Nrf2 transduction or treatment with reduced GSH increased Ager1 expression in AGE-treated primary cells. Downstream, expression of the NRF2 target genes Gstp1 and Hmox1 was also significantly increased following GSH treatment or $\mathrm{Nrf} 2$ transduction compared with expression in Ad-CMV-transduced cells (Supplemental Figure 10).

In patients with NASH and T2DM, RAGE is induced, whereas the clearance receptor AGER1 is significantly downregulated. To assess RAGE and AGER1 expression in patients, we performed RT-qPCR to analyze liver biopsy samples from healthy individuals, patients with steatosis, and patients with NASH and insulin resistance (NASH+IR) or T2DM (NASH+DM) (Figure 6, A and B). We found that RAGE was significantly induced, whereas the clearance receptor AGER1 was reduced in patients with NASH+IR and NASH+DM $(P<0.05)$, but not in those with steatosis only. IHC revealed intense RAGE signal in hepatocytes and macrophages in NASH+DM samples (Figure 6C). Glycosylated hemoglobin (HbA1c) is one of the most studied and clinically used AGEs that is produced by a nonenzymatic reaction between the valine and lysine amino groups of hemoglobin and glucose. Analysis of liver biopsies from a cohort of patients who underwent bariatric surgery revealed that those with histological NASH and abnormal HbA1c $(>5.7)$ had more frequent ductular reaction (DR) and ballooning degeneration on their biopsies (Table 1). In addition, we found that the presence of a DR was associated with lower AGER1 expression levels in the liver (Figure 6D).

\section{Discussion}

In this study, we show that during conditions of high AGEs, an impaired balance of AGER1/ RAGE creates a vicious cycle of enhanced accumulation of AGEs, driving more proinflammatory, oxidative, and profibrogenic conditions in NASH (Figure 7). Although RAGE in macrophages has been shown to have a profound effect on their polarization $(23,24)$, to inhibit cholesterol efflux (25), or to affect inflammasome signaling (26), RAGE in hepatocytes has not been well described. We describe for the first time to our knowledge that RAGE induction in hepatocytes is linked to both NOX4-mediated redox stress pathways and AGER1 downregulation. How these pathways interconnect is important to understand in order to reverse AGE-mediated injury. NOX4, as we previously described, is a major source of oxidative radicals in hepatocytes in NASH (16), and its activation is linked to PKR/ PERK stress pathways and apoptotic cell death. Here, we show that AGEs/RAGE target NOX4 via a SMAD3-dependent pathway and that TGF- $\beta$, a potent transcriptional activator of NOX4, is involved in this process.

An important task is to define the mechanism of AGER1 downregulation in hepatocytes during conditions of high AGEs in NASH. Based on our findings, Rage ${ }^{\mathrm{He} K \mathrm{KO}}$ mice were protected from AGER1 downregulation, and, furthermore, preventing the formation of AGE adducts by PM treatment resulted in increased AGER1 expression. We are aware of the fact that PM may have antioxidant effects; nevertheless, PM showed a direct effect on lowering AGEs, and the composite data from the Rage ${ }^{\text {HepKO }}$ mice suggest a critical role of an AGEs/RAGE-mediated process. Supporting this notion, overexpression of AGER1 in glomerular mesangial cells was able to overcome AGE-mediated inflammatory and oxidative responses in the kidney (27). There are also reports of transgenic AGER1-overexpressing mice (28). These mice have large livers at baseline on a chow diet. As AGER1 is globally induced, AGE uptake and also gut microbiota could be affected, thus this model is less pertinent to NASH pathophysiology, since AGER1 is actually downregulated in patients. We analyzed the potential pathways that are involved in AGER1 downregulation and identified the key role of dysregulated NRF2 responses. Whereas increased NRF2 activity and nuclear translocation are expected during heightened redox activity, we found that NRF2 was paradoxically less active during conditions of high AGEs in hepatocytes. Perturbed NRF2 activity has previously been reported in NASH $(29,30)$, however, the mechanism of inadequate NRF2 responses remains unde- 

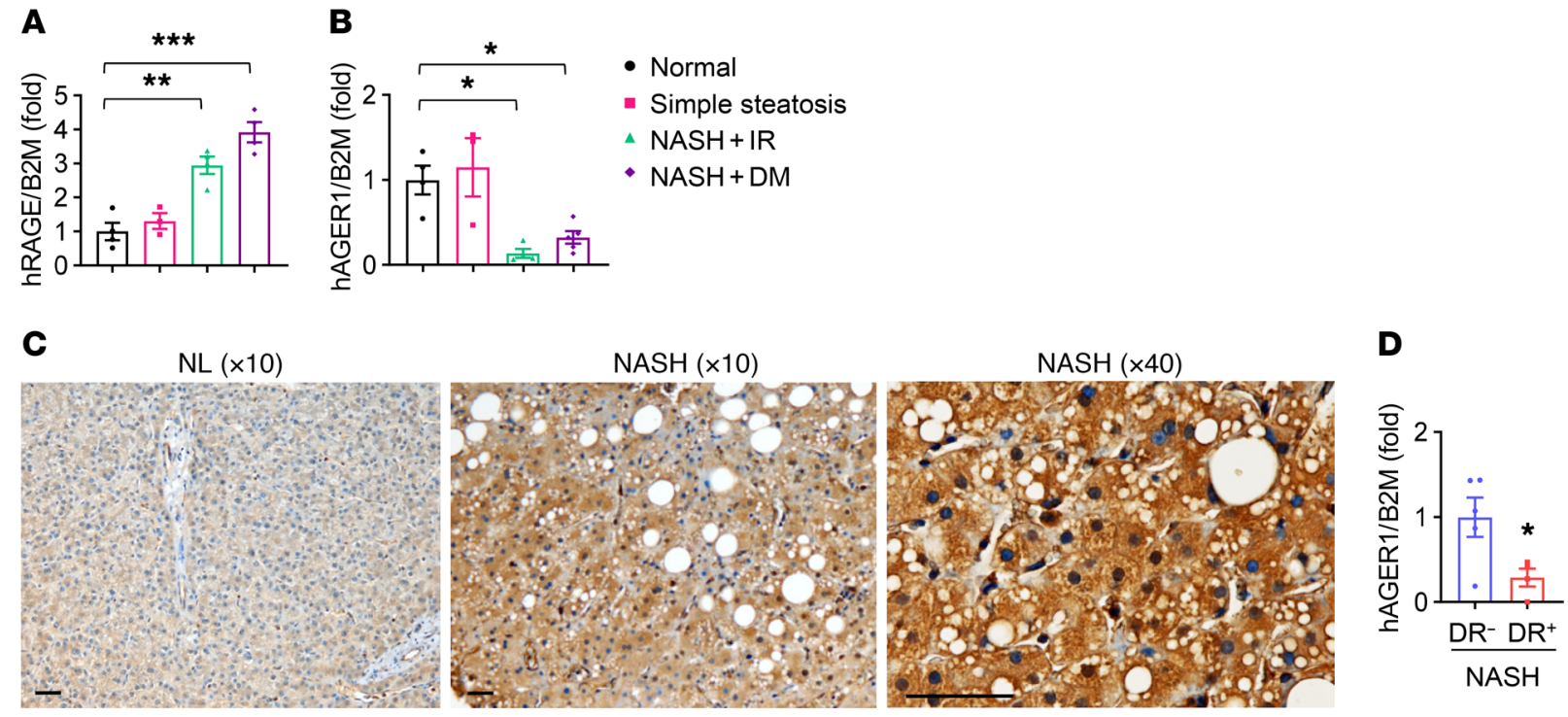

Figure 6. RAGE is induced, whereas AGER1 is reduced, in patients with NASH and IR or T2DM. (A) RT-qPCR analysis of liver biopsy samples from healthy controls, patients with NASH and IR (NASH+IR), and patients with NASH and T2DM (NASH+DM) showed that RAGE was significantly induced in patients with $\mathrm{NASH}+\mathrm{IR}$ and patients with NASH+DM. B2M, $\beta-2$ microglobulin; hRAGE, human RAGE. (B) AGER1 expression was significantly reduced in patients with $\mathrm{NASH}+\mathrm{IR}$ and patients with $\mathrm{NASH}+\mathrm{DM}$. No changes in expression of AGER1 were observed in patients with simple steatosis. (C) A more intense RAGE signal was detected in patients with NASH+DM compared with that in healthy (NL) individuals. Scale bars: $50 \mu \mathrm{m}$. (D) Patients with lower AGER1 levels were more likely to have DR on their biopsies. Additionally, presence of DR and hepatocyte ballooning significantly correlate with serum HbA1c levels (Table 1). Data are presented as the mean \pm SEM. ${ }^{*} P<0.05$, ${ }^{* *} P<0.01$, and ${ }^{* *} P<0.001$, by 1-way ANOVA with Tukey's post hoc test (A and $\left.\mathbf{B}\right)$ or unpaired, 2-tailed $t$ test (E).

fined. First, we show that AAV8-mediated NRF2 transduction in hepatocytes restored AGER1 and improved liver AGEs, inflammation, and fibrosis. Second, we found that NRF2 activity was correlated to the length of the exposure to AGEs. And third, increased neddylation of CUL3, an essential regulatory element of the NRF2 complex, by AGEs rendered NRF2 less stable and destined for degradation. KEAP1, which binds NRF2, is usually able to dissociate from NRF2, allowing its nuclear transport in the face of redox stress. However, cullin neddylation causes conformational changes, and this release cannot occur. The importance of neddylation was recently described in liver fibrosis, in which increased global neddylation was associated with caspase-3 activity and bile acidinduced apoptosis of hepatocytes, and inhibition of neddylation reduced HSC activation (31). It is also interesting to note the paradoxically low NRF2 response accompanied by NOX4 induction in idiopathic pulmonary fibrosis in the context of aging (32). Thus, increased neddylation reactions in the face of increased NOX4 activity could drive inadequate NRF2 and antioxidant responses that in our case affected AGER1.

We also studied AGE-mediated injury and its dependence on NOXs in HSCs and macrophages. Although NOX4 was the dominant target in hepatocytes, in HSCs, both NOX2 and NOX4, and in macrophages, mainly NOX2, played a role in redox activation. However, we could not find evidence of AGE-mediated direct activation of HSCs, consistent with previous reports (33). The paracrine effects of AGE-treated hepatocytes were more prominent; we detected induction of HSC and macrophage activation in a hepatocyte NOX4-dependent manner. ROS play a role in this paracrine communication, however, the role of additional mediators, e.g., extracellular vesicles or damage-associated molecular patterns (DAMPs) cannot be excluded and could be evaluated in future studies.

Expansion of RDCs has been associated with dysregulated regenerative activity and advanced fibrosis in NASH $(15,34)$. We found that in DR was more frequent in both our HiAD model and in patients with T2DM. There is an existing debate as to the exact nature and origin of these cells, but their presence usually signals a more progressive phenotype of NASH. Of note, Pusterla et al. showed that $\mathrm{Mdr2}^{-/-} \mathrm{Rage}^{-/-}$mice developed less hepatocellular carcinoma (HCC) and that progenitor cell expansion was RAGE dependent (35). Furthermore, RAGE was required for expansion of RDCs and tumor promotion in an autophagy-deficient model of HCC (36), and HMGB1, another ligand of RAGE, was involved in progenitor responses and hepatocarcinogenesis (37). In our case,

\section{Table 1. Presence of DR and hepatocyte ballooning correlates with serum HbA1c levels}

\begin{tabular}{ccc} 
& DR & Hepatocellular ballooning \\
HbA1c $<5.7$ & $0(0 / 12)$ & $0.33(4 / 12)$ \\
\hline HbA1c $\geq 5.7$ & $0.33(6 / 18)$ & $0.72(13 / 18)$ \\
& $\chi^{2}=5$ & $\chi^{2}=4.43$ \\
& $P=0.025$ & $P=0.035$
\end{tabular}

Analysis of the liver biopsies and serum samples from a cohort of bariatric surgery patients revealed that the presence of DR and hepatocellular ballooning degeneration were associated with higher serum HbA1c levels. Data were analyzed by $\chi^{2}$ test. $P<0.05$ was considered statistically significant. 


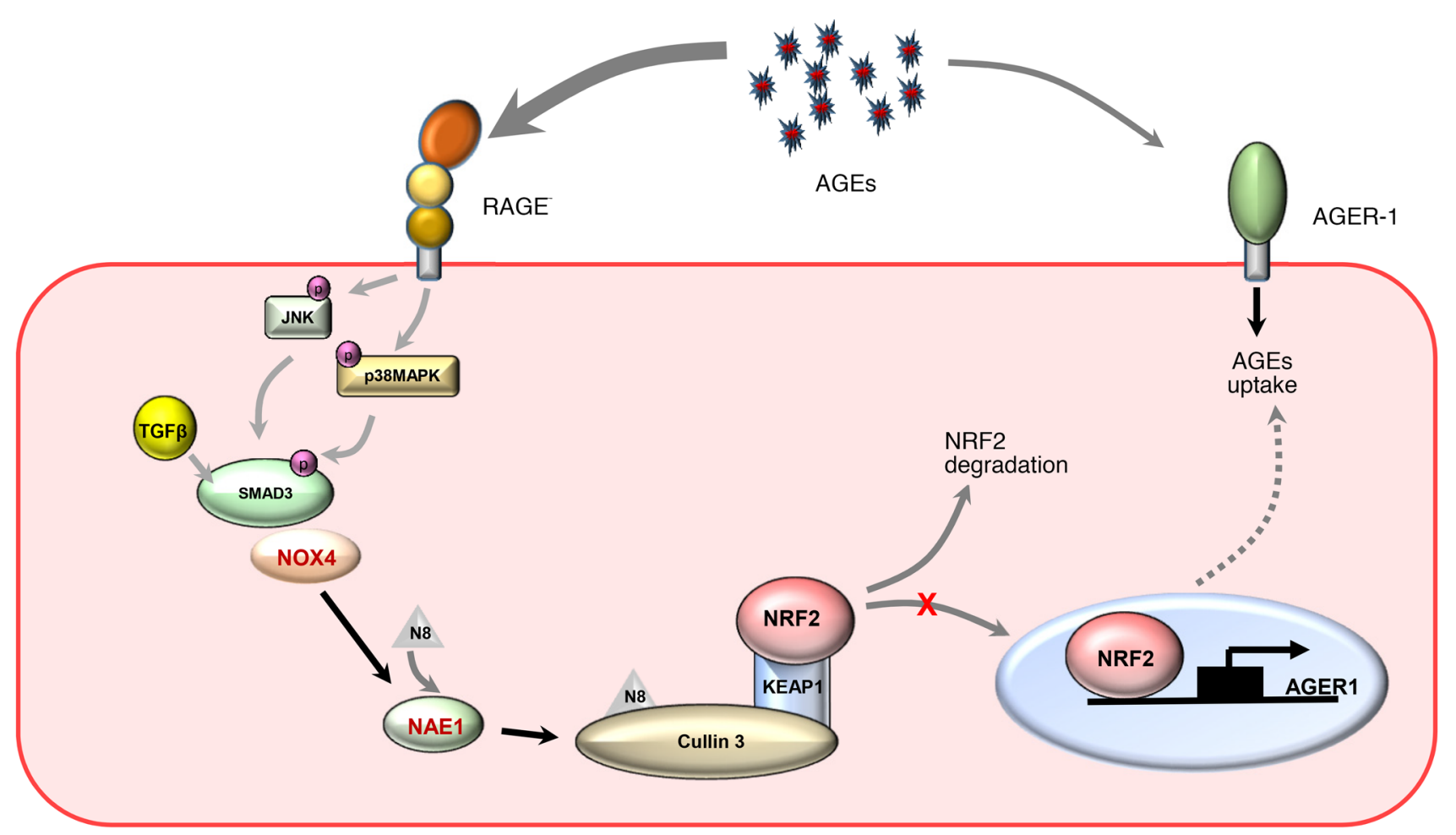

Figure 7. Diagram depicting the mechanism of AGER1 downregulation in hepatocytes. AGEs/RAGE via SMAD3/NOX4 increases neddylation of CUL3, resulting in reduced NRF2 stability. As a consequence, there is a reduction in AGER1 and uptake of AGEs, favoring RAGE-mediated signals.

we deleted RAGE from albumin-expressing cells, thereby including progenitor cells, and, concordant with earlier studies, this may explain why RagetepKO mice had improved DR.

In summary, we show the central role of unbalanced hepatocyte AGER1/RAGE in T2DM and NASH, in which an increased AGEs/RAGE cascade created a redox milieu that was unopposed by NRF2 and its downstream targets. AGER1 downregulation is key to initiating a feed-forward cycle in which further accumulation of AGEs can exacerbate inflammation and fibrosis, even in the absence of significant steatosis (38). Novel therapeutic strategies that target AGER1 will be necessary to prevent NASH and fibrosis progression in patients with T2DM.

\section{Methods}

All primers and antibodies used for this study are listed in Supplemental Tables 2 and 3, respectively.

Human liver tissues. Formalin-fixed, paraffin-embedded (FFPE) liver biopsies were obtained from the UC Davis Cancer Center Biorepository, funded by the National Cancer Institute (NCI). Liver biopsies and matched serum samples from a cohort of 39 patients who underwent bariatric surgery were obtained. Histology was evaluated for necroinflammation, hepatocellular ballooning, DR, and fibrosis by a hepatopathologist in a blinded fashion, and NAS scores are provided.

Animal models. Rage ${ }^{\mathrm{HePKO}}$ were generated by crossing the appropriate $f l / f l$ mice on a C57B6 background (gift from B. Arnold, German Cancer Research Center, Heidelberg, Germany) with albumin-Cre mice for several generations (The Jackson Laboratory). Genotyping with the Cre recombinase primers (forward: GCGGTCTGGCAGTAAAAACTATC; reverse: GTGAAACAGCATTGCTGTCACTT) was performed. Six- to 8-week-old male $f l / f l$, and Rage НерКо littermates were fed either a FFD (catalog 181006, TestDiet), supplemented with $4.2 \%$ high-fructose corn syrup for 14 weeks (16), or, to study the effects of AGEs, a high-AGEs diet (prepared by cooking the FFD at $120^{\circ} \mathrm{C}$ for 20 minutes) as previously described (38), supplemented with highfructose water. A group of littermate control (WT) mice fed a highAGEs diet were injected i.p. daily with PM $\mathrm{HCl}$ (catalog P9158, MilliporeSigma) $(60 \mathrm{mg} / \mathrm{kg}$ ) or vehicle (Tris-HCl) (39). Body weights were monitored daily to adjust the dose.

A group of control $(f l / f l)$ mice fed a high-AGE diet were injected with $5 \times 10^{11}$ genome copies (GC) AAV8 thyroxine-binding globulin (TBG) promoter-Nrf2 or control GFP (Vector Biolabs) via the tail vein at 7 weeks and were then euthanized 7 weeks after injection (14 weeks on the diet). Other groups of Rage $f / / f l$ mice on a high AGEs diet were injected with either AAV6-CMV-Cre $\left(5 \times 10^{11} \mathrm{GC} /\right.$ mouse $)$ or control vectors with no Cre and then sacrificed 7 weeks later. Nox2-KO (B6.129S-Cybbtm1Din/J, JAX) and Nox4-KO mice (B6.129-Nox4tm$1 \mathrm{Kkr} / \mathrm{J}$, JAX) were obtained from The Jackson Laboratory and used for primary cell isolation.

Primary cell isolation, adenoviral transduction, and neddylation experiments. Primary hepatocytes were isolated as previously described (40), and after 24 hours, the cells were transduced with either Ad-Nrf2 or control virus (Applied Biological Materials) with a MOI of 1:50 (2× $10^{5}$ cells were transduced with $10 \times 10^{6} \mathrm{PFU}$ ) for 24 hours. Cells were treated with AGEs-BSA or BSA with or without reduced GSH (10 mM) (CAS 70-18-8, catalog 3541, MilliporeSigma) 30 minutes before AGEsBSA treatment. To analyze Nae1 expression, primary WT hepatocytes were serum starved and transduced with Ad-Nox4 (Applied Biological Materials) or with the control vector Ad-CMV with a MOI of 1:50 
for 48 hours. After 48 hours, the cells were treated with $100 \mathrm{mg} / \mathrm{mL}$ AGEs or control BSA for 30 minutes. For neddylation inhibition analysis, primary WT hepatocytes were treated with $3 \mu \mathrm{M}$ NAE1 inhibitor MLN4924 (catalog 5054770001, MilliporeSigma) and AGEs (or control BSA) for 24 hours. At the end of the experiments, mRNA was extracted and reverse transcribed, and RT-qPCR was performed as described below.

Primary stellate cells were isolated from mice as described previously (41) and cultured in Medium 199 (MilliporeSigma) with 20\% FBS (Gibco, Thermo Fisher Scientific) and antibiotics. Liver macrophages were isolated as previously described (42) and cultured in RPMI (Gibco, Thermo Fisher Scientific) with 10\% FBS and antibiotics.

Preparation of AGEs-BSA. AGEs-BSA were prepared as previously described (43, 44). Briefly, glycolaldehyde (catalog G6805, MilliporeSigma) was dissolved in $10 \mathrm{mg} / \mathrm{mL}$ BSA in PBS to a final concentration of $33 \mathrm{mM}$. The solutions were incubated at $37^{\circ} \mathrm{C}$ for 72 hours followed by dialysis against PBS. The dialyzed solutions were sterilized with $2-\mu \mathrm{M}$ filters, and aliquots were stored at $-80^{\circ} \mathrm{C}$. Cells were treated with $100 \mu \mathrm{g} / \mathrm{mL}$ AGEs-BSA, and $100 \mu \mathrm{g} / \mathrm{mL}$ BSA in PBS was used as a control.

Measurement of AGEs content. The amount of AGEs was measured using a OxiSelect Advanced Glycation End Product Competitive ELISA Kit (Cell Biolabs) in the serum and the liver homogenate, according to the manufacturer's instructions. Briefly, 10-mg liver samples were homogenized in PBS. After measuring the protein concentration, 300 $\mu \mathrm{g}$ protein or $50-\mu \mathrm{L}$ serum samples were added to a 96-well ELISA plate and incubated for 1 hour at room temperature. Following incubation with the secondary HRP-conjugated anti-AGEs, the reaction was halted with a stop solution and the plates were read at $450 \mathrm{~nm}$. To measure AGEs in the diet, 1 gram of chow, FFD, or HiAD was homogenized in PBS and then centrifuged at $1000 \times g$ for 10 minutes at $4^{\circ} \mathrm{C}$. AGEs were measured by the OxiSelect AGEs Competitive ELISA Kit according to the manufacturer's protocol. AGE concentrations were normalized to the protein amount in each diet. The AGEs content was measured in 3 different batches, and the data are presented as the fold difference between the diets. The amount of protein, fat, sugar, and fiber in the HiAD was determined by Eurofins Scientific.

$m R N A$ extraction, reverse transcription, and RT-qPCR. Total RNA was isolated from the human FFPE liver samples using RecoverAll Total Nucleic Acid Isolation (Invitrogen, Thermo Fisher Scientific) and from fresh-frozen mouse liver tissues or cells using the RNeasy Kit (QIAGEN). cDNA was reverse transcribed from $1 \mu \mathrm{g}$ mRNA using the iScriptTM cDNA Synthesis Kit (Bio-Rad). RT-qPCR was performed using Power SYBR Green PCR Master Mix (Applied Biosystems, Thermo Fisher Scientific) on a 7900HT Fast Real-Time PCR System (Thermo Fisher Scientific). Relative concentrations were normalized to the housekeeping genes.

Hydroxyproline assay. The hydroxyproline assay was performed as previously described (16). Briefly, liver samples were homogenized and denatured in $6 \mathrm{~N} \mathrm{HCl}$. Hydrolyzed samples were then dried and washed 3 times with deionized water followed by incubation in $50 \mathrm{mM}$ chloramine $\mathrm{T}$ oxidation buffer for 20 minutes at room temperature. The samples were then incubated with $3.15 \mathrm{M}$ perchloric acid (catalog 311421, MilliporeSigma) for 5 minutes, and then with p-dimethylaminobenzaldehyde (catalog D2004, MilliporeSigma). Absorbance of each sample was measured at $557 \mathrm{~nm}$, and values were normalized to each sample's weight before hydrolysis.
IHC. IHC was performed as previously described (16). Paraffinembedded slides were deparaffinized with 2 changes of sylene and 3 changes of $100 \%, 95 \%$, and $75 \%$ ethanol. Antigen retrieval was carried out by boiling the slides in citrate buffer ( $\mathrm{pH}$ 6.0) for 12 minutes in a microwave oven on high power. Following blocking with $5 \%$ goat serum, the slides were incubated overnight with the primary antibody at $4^{\circ} \mathrm{C}$. After 3 washes, the slides were incubated with the appropriate biotinylated secondary antibody using the ABC Peroxidase Standard Staining Kit (Thermo Fisher Scientific). The slides were then stained with DAB (Abcam) and counterstained with hematoxylin (Thermo Fisher Scientific). Images were taken with a KEYENCE BZX-800 microscope or a Leica DM2000. The number of CK19+SOX9+ cells was counted in 5 different fields in 3-4 mice/group.

PSR. Paraffin-embedded liver sections were deparaffinized and hydrated. The slides were placed in $0.2 \%$ phosphomolybdic acid (Thermo Fisher Scientific) for 5 minutes and rinsed in deionized water, stained with PSR solution (Polysciences) for 1 hour, and then washed twice with $0.5 \%$ acetic acid for 1 minute, dehydrated twice in $100 \%$ ethanol for 1 minute, cleared in xylene, and then mounted. The slides were scanned with a Leica Aperio AT2 at the Stanford Human Pathology-Histology Service Center. ImageJ software (NIH) (45) was used to quantify the positively stained areas. Four random fields $(\times 20$ magnification) for 3-4 mice per group were quantified.

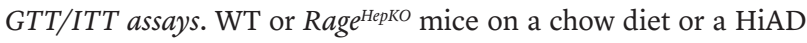
were subjected to GTT/ITT assays. Following overnight fasting, the GTT was initiated with an i.p. injection of $2 \mathrm{~g} / \mathrm{kg}$ glucose (MilliporeSigma). The results were read by Germaine Laboratories AimStrip Plus at the indicated time points $(0,15,30,60$, and 120 minutes). An ITT was conducted in the mice after a 4 - hour fast followed by and injection of $1 \mathrm{mUI} / \mathrm{g}$ i.p. insulin (Humulin R, Lilly). There was at least a 1-week interval between the GTT and ITT assays and a 48-hour interval between the GTT and euthanization.

Transwell coculture assays. Primary hepatocytes from WT and Nox4KO mice were isolated and cultured in the lower chambers. The 2 chambers were separated by a semipermeable membrane with a pore size of $0.4 \mu \mathrm{m}$ (Transwell culture plates, BD Biosciences). After 24 hours of serum starvation, primary hepatocytes were treated with AGEs-BSA or BSA overnight. Cells were then washed with PBS, and fresh culture medium was added with or without GSH $(10 \mathrm{mM})$. Macrophages and HSCs isolated from WT mice were placed in the upper chamber of the Transwell units. After 24 hours of coculturing, RT-qPCR was performed to analyze mRNA expression in macrophages and HSCs.

Detection of CUL3 neddylation. Liver tissue samples were homogenized in RIPA buffer (10 mM Tris-HCl [pH 8.0], 1 mM EDTA, 0.5 mM EGTA, 1\% Triton X-100, 0.1\% sodium deoxycholate, 0.1\% SDS, $140 \mathrm{mM} \mathrm{NaCl}$ ), supplemented with cOmplete Protease Inhibitor Cocktail (Roche). HepG2 cells (American Type Culture Collection) were cultured in DMEM (Gibco, Thermo Fisher Scientific, 11995065) supplemented with 10\% FBS (Gibco, Thermo Fisher Scientific) and penicillin/streptomycin. Cells were treated with AGEs or BSA for the indicated durations and lysed with RIPA buffer. Liver homogenates or cell lysates were subjected to 7.5\% SDS PAGE gel and transferred onto a nitrocellulose membrane and then incubated overnight with antiCUL3 rabbit polyclonal antibody (Cell Signaling Technology, 2759). Following secondary antibody incubation, the membranes were developed by ECL (Advansta). Neddylation of CUL3 was detected by the presence of a double band at approximately $80 \mathrm{kDa}(46,47)$. 
Extraction of nuclear fractions. Nuclear fractions were extracted with NE-PER Nuclear and Cytoplasmic Extraction Reagents (Thermo Fisher Scientific) following the manufacturer's protocol. Cells were collected in PBS with protease inhibitor, centrifuged at $4^{\circ} \mathrm{C}$, and lysed in a cytoplasmic extraction reagent that was removed by centrifugations at $10,000 \times g$ at $4^{\circ} \mathrm{C}$. The pellet was resuspended in the nuclear extraction reagent, and the nuclear fraction was collected.

ChIP assay. A ChIP assay was conducted on the liver samples using the EZ-Magna ChIP HiSens (MilliporeSigma) according to the manufacturer's protocol. Liver samples were cut into pieces of approximately $5 \mathrm{~mm}$ in diameter and washed in cold PBS. Crosslinking was performed in $1 \%$ formaldehyde. The reaction was then terminated with $125 \mathrm{mM}$ glycine. After homogenization, the cells were lysed in nuclei isolation buffer supplemented with a protease inhibitor. To sheer DNA, isolated chromatins were sonicated on wet ice. Using ChIP A/G magnetic beads, cross-linked protein-DNA was then immunoprecipitated with anti-SMAD3 antibody. After elution, DNA was purified and analyzed with RT-qPCR using the following primers: forward, $5^{\prime}$-TTGTGTATGTGTGTGTGTGTGTG-3' and reverse, 5'-AAATGTGGTAGAGAACAATCAAGAA-3'. The amplicon (-2549 to -2492$)$ contains a putative SMAD3-binding domain (-2540 to -2531) and is adjacent to another at -2429 to -2420 . The data were analyzed with $\Delta \Delta \mathrm{Ct}$ values normalized to the input. Negative control primers (provided in the kit) were tested following the instructions, and no signal was detected.

Lucigenin assay. After tissue homogenization or cell lysis in sucrose buffer, the membrane fractions were extracted by centrifugation at $100,000 \times g$ for 1 hour. The pellet was resuspended in Krebs buffer and incubated at $37^{\circ} \mathrm{C}$ for 30 minutes. After incubation with lucigenin buffer (Thermo Fisher Scientific), $100 \mu \mathrm{M}$ NADPH was added to each sample, and the chemiluminescence intensity was measured by a luminometer (Monolight Luminometer) every minute for 10 minutes. The data were normalized to the protein content of each sample.

Luciferase reporter assay. Primary hepatocytes were transfected with pGL-hNOX4-Luc (a gift from C. Yabe, Kyoto University, Kyoto, Japan) by the JetPEI Reagent (Polyplus Transfection) according to the manufacturer's protocol. Cells were treated with $100 \mu \mathrm{g} / \mathrm{mL}$ AGEs, and after 24 hours, the reporter assay was performed with the Promega Luciferase Assay System. Data were normalized to the protein concentration of each sample. For analysis of AGER1 promoter activity, HepG2 cells were cultured in DMEM supplemented with 10\% FBS. Following 3-5 passages, Lipofectamine 3000 (Thermo Fisher Scientific) was used to transfect the cells with AGER1 (dolichyl-diphosphooligonucleotidesaccharid--protein glycosyltransferase noncatalytic subunit $[D D O S T])$ from the Promoter Reporter GoClone Collection (Active Motif, product ID: S707468) for 72 hours. The cells were transduced with the NRF2 (or control CMV) adenoviral vector system (Applied Biological Materials) at a MOI of 1:50 for 24 hours. Following overnight starvation, the cells were treated with $100 \mu \mathrm{g}$ BSA or
AGEs for 24 hours. AGER1 promoter activity was measured using the LightSwitch Luciferase Assay Kit (Active Motif) following the manufacturer's protocol. The values were normalized to the protein content.

NRF2 stability assays. Primary WT hepatocytes were treated with AGEs (or control BSA) for 24 hours and then washed twice with PBS and incubated with $0.5 \mu \mathrm{g} / \mathrm{mL}$ cycloheximide (MilliporeSigma) for the indicated durations. Cell lysates were separated with SDS-PAGE gel and transferred onto PVDF membranes. The membranes were incubated overnight with a NRF2 primary antibody and then with the appropriate secondary antibody.

Statistics. Data are presented as the mean \pm SEM. A 1-way ANOVA followed by a 2-tailed, unpaired Student's $t$ test were performed to analyze differences between groups. Comparisons between 3 or more groups were analyzed using a 1-way ANOVA with Tukey's post hoc test for multiple comparisons for parametric data. A $\chi^{2}$ test was performed to evaluate the correlation between variables. A $P$ value of less than 0.05 was considered statistically significant. Data analysis and the graphs were done using GraphPad Prism 8 (GraphPad Software). Analysis of outliers was done with the ROUT (robust regression followed by outlier identification) test $(Q=1 \%)$.

Study approval. Experiments involving patient samples were approved by the IRB of the UC Davis Medical Center. All patients provided written informed consent. Animal experiments were performed according to the experimental procedures approved by the IACUCs of the University of California Davis and the VA Palo Alto.

\section{Author contributions}

$\mathrm{AD}$ acquired, analyzed, and interpreted data. WF, SRF, SD, GM, and MA acquired data. JXJ and KAW acquired and analyzed data. KAO and GWC interpreted data. YL performed statistical analysis and wrote the manuscript. NJT conceptualized, designed, and supervised the study, analyzed data, wrote the manuscript, and acquired funding.

\section{Acknowledgments}

We thank Parul Gupta, Hyesuk Park, and Sutapa Sarkar (all from Stanford University) and Judit Lopez (UC Davis) for their technical help with the studies. This research was supported by funding from the National Institute of Diabetes and Digestive and Kidney Diseases (NIDDK), NIH (R01 2DK083283, to NJT); the National Institute on Aging (NIA), NIH (1R01AG060726, to NJT); the SPARK Program (Stanford University); and the VA (IO1 BX002418, to NJT).

Address correspondence to: Natalie J. Török, Division of Gastroenterology and Hepatology, Stanford School of Medicine, 300 Pasteur Alway Building Room M207, Stanford, California 94304, USA. Phone: 650.723.2188; Email: ntorok@stanford.edu.
1. Anstee QM, Targher G, Day CP. Progression of NAFLD to diabetes mellitus, cardiovascular disease or cirrhosis. Nat Rev Gastroenterol Hepatol. 2013;10(6):330-344.

2. Bazick J, et al. Clinical model for NASH and advanced fibrosis in adult patients with diabetes and NAFLD: guidelines for referral in NAFLD. Diabetes Care. 2015;38(7):1347-1355.

3. Brownlee M. Biochemistry and molecular cell biology of diabetic complications. Nature. 2001;414(6865):813-820.

4. Vlassara H, Uribarri J. Advanced glycation end products (AGE) and diabetes: cause, effect, or both? Curr Diab Rep. 2014;14(1):453.

5. Henning C, Smuda M, Girndt M, Ulrich C, Glomb MA. Molecular basis of maillard amide-advanced glycation end product (AGE) formation in vivo. J Biol Chem. 2011;286(52):44350-44356.
6. Colhoun HM, et al. Total soluble and endogenous secretory receptor for advanced glycation end products as predictive biomarkers of coronary heart disease risk in patients with type 2 diabetes: an analysis from the CARDS trial. Diabetes. 2011;60(9):2379-2385

7. McVicar CM, et al. Role of the receptor for advanced glycation endproducts (RAGE) in retinal vasodegenerative pathology during diabetes 
in mice. Diabetologia. 2015;58(5):1129-1137.

8. Li YM, et al. Molecular identity and cellular distribution of advanced glycation endproduct receptors: relationship of p60 to OST-48 and p90 to $80 \mathrm{~K}-\mathrm{H}$ membrane proteins. Proc Natl Acad Sci USA. 1996;93(20):11047-11052.

9. Ott C, Jacobs K, Haucke E, Navarrete Santos A, Grune T, Simm A. Role of advanced glycation end products in cellular signaling. Redox Biol. 2014;2:411-429.

10. Wautier MP, Chappey O, Corda S, Stern DM, Schmidt AM, Wautier JL. Activation of NADPH oxidase by AGE links oxidant stress to altered gene expression via RAGE. Am J Physiol Endocrinol Metab. 2001;280(5):E685-E694.

11. Krishnan A, et al. A longitudinal study of whole body, tissue, and cellular physiology in a mouse model of fibrosing NASH with high fidelity to the human condition. Am J Physiol Gastrointest Liver Physiol. 2017;312(6):G666-G680.

12. Chen JL, Francis J. Pyridoxamine, advanced glycation inhibition, and diabetic nephropathy. J Am Soc Nephrol. 2012;23(1):6-8.

13. Turgut F, Bolton WK. Potential new therapeutic agents for diabetic kidney disease. Am J Kidney Dis. 2010;55(5):928-940.

14. Zhao L, Westerhoff M, Pai RK, Choi WT, Gao ZH, Hart J. Centrilobular ductular reaction correlates with fibrosis stage and fibrosis progression in non-alcoholic steatohepatitis. Mod Pathol. 2018;31(1):150-159.

15. Machado MV, et al. Accumulation of duct cells with activated YAP parallels fibrosis progression in non-alcoholic fatty liver disease. J Hepatol. 2015;63(4):962-970.

16. Bettaieb A, et al. Hepatocyte nicotinamide adenine dinucleotide phosphate reduced oxidase 4 regulates stress signaling, fibrosis, and insulin sensitivity during development of steatohepatitis in mice. Gastroenterology. 2015;149(2):468-80.e10.

17. Rezvani $\mathrm{M}$, et al. In vivo hepatic reprogramming of myofibroblasts with AAV vectors as a therapeutic strategy for liver fibrosis. Cell Stem Cell. 2016;18(6):809-816

18. Messeguer X, Escudero R, Farré D, Núñez O, Martínez J, Albà MM. PROMO: detection of known transcription regulatory elements using species-tailored searches. Bioinformatics. 2002;18(2):333-334.

19. Hayes JD, Dinkova-Kostova AT. The Nrf2 regulatory network provides an interface between redox and intermediary metabolism. Trends Biochem Sci. 2014;39(4):199-218.
20. Calkins MJ, et al. The Nrf2/ARE pathway as a potential therapeutic target in neurodegenerative disease. Antioxid Redox Signal. 2009;11(3):497-508.

21. Tan Y, et al. Diabetic downregulation of Nrf2 activity via ERK contributes to oxidative stress-induced insulin resistance in cardiac cells in vitro and in vivo. Diabetes. 2011;60(2):625-633.

22. Niture SK, Khatri R, Jaiswal AK. Regulation of Nrf2an update. Free Radic Biol Med. 2014;66:36-44.

23. Rojas A, Añazco C, Araya P. M2 macrophages do not fly into a "RAGE”. Inflamm Res. 2017;66(1):13-15.

24. Son M, et al. C1q and HMGB1 reciprocally regulate human macrophage polarization. Blood. 2016;128(18):2218-2228.

25. Daffu G, et al. RAGE suppresses ABCG1-mediated macrophage cholesterol efflux in diabetes. Diabetes. 2015;64(12):4046-4060.

26. Son S, Hwang I, Han SH, Shin JS, Shin OS, Yu JW. Advanced glycation end products impair NLRP3 inflammasome-mediated innate immune responses in macrophages. J Biol Chem. 2017;292(50):20437-20448.

27. Lu C, He JC, Cai W, Liu H, Zhu L, Vlassara H. Advanced glycation endproduct (AGE) receptor 1 is a negative regulator of the inflammatory response to AGE in mesangial cells. Proc Natl Acad Sci USA. 2004;101(32):11767-11772.

28. Zhuang A, et al. Increased liver AGEs induce hepatic injury mediated through an OST48 pathway. Sci Rep. 2017;7(1):12292.

29. Ka SO, Bang IH, Bae EJ, Park BH. Hepatocyte-specific sirtuin 6 deletion predisposes to nonalcoholic steatohepatitis by up-regulation of Bach1, an Nrf2 repressor. FASEB J. 2017;31(9):3999-4010.

30. Lee DH, et al. Ezetimibe, an NPC1L1 inhibitor, is a potent Nrf2 activator that protects mice from diet-induced nonalcoholic steatohepatitis. Free Radic Biol Med. 2016;99:520-532.

31. Zubiete-Franco I, et al. Deregulated neddylation in liver fibrosis. Hepatology. 2017;65(2):694-709.

32. Hecker L, et al. Reversal of persistent fibrosis in aging by targeting Nox4-Nrf2 redox imbalance. Sci Transl Med. 2014;6(231):231ra47.

33. Lohwasser C, Neureiter D, Popov Y, Bauer M, Schuppan D. Role of the receptor for advanced glycation end products in hepatic fibrosis. World J Gastroenterol. 2009;15(46):5789-5798.

34. Gadd VL, et al. The portal inflammatory infiltrate and ductular reaction in human nonalcoholic fatty liver disease. Hepatology. 2014;59(4):1393-1405.
35. Pusterla T, et al. Receptor for advanced glycation endproducts (RAGE) is a key regulator of oval cell activation and inflammation-associated liver carcinogenesis in mice. Hepatology. 2013;58(1):363-373.

36. Khambu B, et al. HMGB1 promotes ductular reaction and tumorigenesis in autophagy-deficient livers. JClin Invest. 2018;128(6):2419-2435.

37. Hernandez C, Huebener P, Pradere JP, Antoine DJ, Friedman RA, Schwabe RF. HMGB1 links chronic liver injury to progenitor responses and hepatocarcinogenesis. J Clin Invest. 2018;128(6):2436-2451.

38. Patel R, et al. Effect of dietary advanced glycation end products on mouse liver. PLoS One. 2012;7(4):e35143.

39. Muellenbach EA, et al. Interactions of the advanced glycation end product inhibitor pyridoxamine and the antioxidant alpha-lipoic acid on insulin resistance in the obese Zucker rat. Metab Clin Exp. 2008;57(10):1465-1472.

40. Li WC, Ralphs KL, Tosh D. Isolation and culture of adult mouse hepatocytes. Methods Mol Biol. 2010;633:185-196.

41. Geerts A, et al. Purification of rat hepatic stellate cells by side scatter-activated cell sorting. Hepatology. 1998;27(2):590-598.

42. Zigmond $\mathrm{E}$, et al. Infiltrating monocyte-derived macrophages and resident kupffer cells display different ontogeny and functions in acute liver injury. J Immunol. 2014;193(1):344-353.

43. Unno Y, et al. Glycolaldehyde-modified bovine serum albumin downregulates leptin expression in mouse adipocytes via a CD36-mediated pathway. Ann N Y Acad Sci. 2005;1043:696-701.

44. Guimarães EL, Empsen C, Geerts A, van Grunsven LA. Advanced glycation end products induce production of reactive oxygen species via the activation of NADPH oxidase in murine hepatic stellate cells. J Hepatol. 2010;52(3):389-397.

45. Schindelin J, Rueden CT, Hiner MC, Eliceiri KW. The Image J ecosystem: An open platform for biomedical image analysis. Mol Reprod Dev. 2015;82(7-8):518-529.

46. Zhou W, et al. UBE2M is a stress-inducible dual E2 for neddylation and ubiquitylation that promotes targeted degradation of UBE2F. Mol Cell. 2018;70(6):1008-1024.e6.

47. Zhou $\mathrm{H}$, et al. A potent small-molecule inhibitor of the DCN1-UBC12 interaction that selectively blocks cullin 3 neddylation. Nat Commun. 2017;8(1):1150. 